\title{
Evidence on the effectiveness of small unmanned aircraft systems (sUAS) as a survey tool for North American terrestrial, vertebrate animals: a systematic map protocol
}

Jared A. Elmore ${ }^{1 *} \mathbb{D}$, Michael F. Curran ${ }^{1}$, Kristine O. Evans' ${ }^{1}$, Sathishkumar Samiappan², Meilun Zhou², Morgan B. Pfeiffer ${ }^{3}$, Bradley F. Blackwell ${ }^{3}$ and Raymond B. Iglay ${ }^{1}$

\begin{abstract}
Background: Small unmanned aircraft systems (SUAS) are replacing or supplementing manned aircraft and groundbased surveys in many animal monitoring situations due to better coverage at finer spatial and temporal resolutions, access, cost, bias, impacts, safety, efficiency, and logistical benefits. Various sUAS models and sensors are available with varying features and usefulness depending on survey goals. However, justification for selection of sUAS and sensors are not typically offered in published literature and existing reviews do not adequately cover past and current sUAS applications for animal monitoring nor their associated sUAS model and sensor technologies, taxonomic and geographic scope, flight conditions and considerations, spatial distributions of sUAS applications, and reported technical difficulties. We outline a systematic map protocol to collect and consolidate evidence pertaining to sUAS monitoring of animals. Our systematic map will provide a useful synthesis of current applications of sUAS-animal related studies and identify major knowledge clusters (well-represented subtopics that are amenable to full synthesis by a systematic review) and gaps (unreported or underrepresented topics that warrant additional primary research) that may influence future research directions and sUAS applications.

Methods: Our systematic map will investigate the current state of knowledge using an accurate, comprehensive, and repeatable search. We will find relevant peer-reviewed and grey literature as well as dissertations and theses using online publication databases, Google Scholar, and by request through a professional network of collaborators and publicly available websites. We will use a tiered approach to article exclusion with eligible studies being those that monitor (i.e., identify, count, estimate, etc.) terrestrial vertebrate animals. Extracted data concerning sUAS, sensors, animals, methodology, and results will be recorded in Microsoft Access. We will query and catalogue evidence in the final database to produce tables, figures, and geographic maps to accompany a full narrative review that answers our primary and secondary questions.
\end{abstract}

Keywords: Count, Drone, Monitor, RPA, UAV, UVS, Wildlife

*Correspondence: jae133@msstate.edu

${ }^{1}$ Department of Wildlife, Fisheries and Aquaculture, Mississippi State University, Thompson Hall, Mississippi State, Mississippi 39762, USA

Full list of author information is available at the end of the article

\section{Background}

Small unmanned aircraft systems [sUAS, also commonly referred to as unmanned aerial vehicle (UAV), unmanned vehicle system (UVS), unmanned aircraft (UA), remotely piloted aircraft (RPA), or drones], are defined as powered aircraft controlled either remote or by automatic

(c) The Author(s) 2021. This article is licensed under a Creative Commons Attribution 4.0 International License, which permits use, sharing, adaptation, distribution and reproduction in any medium or format, as long as you give appropriate credit to the original author(s) and the source, provide a link to the Creative Commons licence, and indicate if changes were made. The images or other third party material in this article are included in the article's Creative Commons licence, unless indicated otherwise in a credit line to the material. If material is not included in the article's Creative Commons licence and your intended use is not permitted by statutory regulation or exceeds the permitted use, you will need to obtain permission directly from the copyright holder. To view a copy of this licence, visit http://creativeco mmons.org/licenses/by/4.0/. The Creative Commons Public Domain Dedication waiver (http://creativecommons.org/publicdomain/ zero/1.0/) applies to the data made available in this article, unless otherwise stated in a credit line to the data. 
programming, weighing less than $24.9 \mathrm{~kg}$, and without an onboard human pilot $[1,2]$. In many situations, sUASs are replacing or supplementing manned aircraft and ground based surveys as they have demonstrated promise for developing automated and standardized animal assessments from digital aerial surveys [3-6]. Primary benefits of using sUASs for animal surveys include their ability to cover expansive areas at fine spatial and temporal resolutions, reduce surveyor bias and labor costs, access inconvenient locations, minimize environmental impacts including problematic animal behavior, and increased personnel safety and logistical operations compared to manned aircraft [4, 5, 7-15].

To date, sUAS have been used for a wide variety of animal assessments including wildlife and domestic animals. Monitoring efforts include counts for abundance or richness, behavioral studies, location, movement tracking, and habitat quality assessments for a diverse array of taxa [4] including birds (e.g., [5, 15-18]), mammals (e.g., [1922]), and reptiles (e.g., [23]) in both marine and terrestrial systems. Multiple commercial sUAS models and sensors are available and vary in their usefulness by survey goals and situations $[10,16]$. For example, sUAS models (e.g., DJI Matrice 600 Pro) may be chosen based on platform type (e.g., fixed-wing or multirotor), ease of use, payload capacity to carry the desired sensor, or cost [4], and sensors may be chosen based on what type of remotely sensed data are desired and survey conditions (e.g., optical sensors for determining animal behavior; see [24]; or thermal sensors for detecting cryptic nocturnal animals; see [25]). 'Off-the-shelf' sUAS packages further support widespread use of sUAS and exploration of new applications [16]. However, recent rapid increases in sUAS use, improvements to associated model, sensor, and computer vision technologies, and a common interest to incorporate sUAS in myriad situations have stemmed from a relatively sparse foundation of scientific investigations, common approaches and limited shared knowledge of operating guidelines to effectively incorporate this new technology. For example, justifications for selecting particular sUAS models and sensors are typically not offered in studies, and standard reporting mechanisms have only recently emerged [26]. Further confounding the use of sUAS for animals counts are a lack of bias corrected estimates (i.e., estimates that account for sampling errors such as not detecting the presence of an individual or double counting individuals), despite calls for such studies [27]. Bias-corrected estimates are important to determining best practices for using sUAS as an effective and reliable animal monitoring tool as they allow accurate estimates of animal populations [27, 28].

Systematic maps aim to answer broader questions than systematic reviews by collating, describing, and cataloging evidence related to the topic of interest [29] while following reporting standards for systematic evidence synthesis (see Additional file 1 for ROSES checklist). A preliminary literature search returned no existing systematic maps pertaining to this issue, and yet, a systematic map would set the necessary foundation for an explosion of scientific rigor supporting sUAS applications in animal monitoring. Most published reviews focus on future sUAS use [10], overall accomplishments and challenges [5, 6], animal behavioral responses [30], or general research summaries $[4,17]$. A systematic map, however, would at minimum, allow evaluation and summarization of past and current sUAS applications for animal monitoring among sUAS model and sensor technologies, taxonomic and geographic scope, flight conditions and operational considerations, spatial distributions of sUAS applications, and reported technical benefits and pitfalls. From the generated body of evidence, standard reporting mechanisms, selection criteria, and use applications could be revealed to complement recent efforts (e.g., [26]) and investigate potential bias associated with sUAS animal surveys.

Our objective is to develop a systematic map to consolidate evidence in the aforementioned areas affecting and pertaining to use of sUASs to monitor animals in terrestrial environments. Given the rapid expansion of sUAS technology, we envision this systematic map informing future researchers, practitioners and other end users planning to use sUAS in terrestrial ecosystems to monitor animals, especially wild animals, of the best practices based on the current state of knowledge. While much literature has been published in marine systems, this systematic map aims to focus on vertebrates in terrestrial systems only, and primarily those of North America, due to interest of collaborators, stakeholders, and funders whose overall goal involves understanding and mitigating terrestrial animal-vehicle collisions [31,32]. The systematic map will also elucidate major knowledge clusters (well-represented subtopics that are amenable to full synthesis by a systematic review) and gaps (unreported or underrepresented topics that warrant additional primary research) among sUAS-animal related studies, as well as summarizing current applications, as previously mentioned, and a repeatable framework for future sUAS assessments.

This protocol was developed in collaboration with Mississippi State University Department of Wildlife, Fisheries and Aquaculture (MSU-WFA), the United States Department of Agriculture Animal and Plant Health Inspection Service, Wildlife Services, National Wildlife Research Center (USDA-APHIS-WS-NWRC), and the Federal Aviation Administration (FAA). These three entities are all considered stakeholders. Authors 
are members of these entities with the exception of the FAA, whose senior members reviewed this systematic map protocol. The main authors discussed and refined the scope and objective of this systematic map during initial project planning meetings. The findings of this systematic map will be of direct interest to each of these stakeholders in addition to numerous other stakeholders in academia, research, industry, and other government agencies.

\section{Objective of the review}

The objective of this review is to determine the current state of knowledge regarding how sUAS have been used to monitor terrestrial, vertebrate animals. While sUAS have been used for automated and standardized animal assessments using digital aerial surveys, the extent to which they have been used has not been fully summarized, including study specifics such as effectiveness and accuracy. This review will provide a quantitative, repeatable process to develop objective selection criteria and rank sUAS models and sensors for monitoring and counting animals in terrestrial environments. Our specific goal is to provide a comprehensive, catalogued state of knowledge surrounding sUAS models and sensors used to monitor terrestrial animals.

\section{Primary question}

What evidence exists on the effectiveness of sUAS as a survey tool for terrestrial, vertebrate animals?

\section{Secondary questions}

1. What sUAS models and sensors are most used?

2. What are the common statistical approaches and field methodologies of sUAS applications for monitoring animals?

3. Do sUAS applications for monitoring animals differ among geographic ranges, habitat types (i.e., land covers), species or species groups, survey models, or survey environments?

4. What factors affect accuracy (i.e., sampling bias) in sUAS imagery (e.g., animal size, behavior, land/water cover, weather or light conditions, etc.)?

5. What are the common constraints of sUAS for monitoring animals (e.g., sUAS models and sensors, government restrictions, sensor calibration, expense, battery life, etc.)?

6. What are the suggested statistical approaches and characteristics of sampling designs that can lead to a consistent set of best practices to avoid, reduce, and correct sampling bias from sUAS aerial imagery while still achieving project goals?

\section{Components of the primary question}

Population (P): all terrestrial vertebrate wildlife species and domestic animals (inclusive of wildlife that also commonly occur in aquatic systems but are typically referred to as terrestrial such as shorebirds, waterfowl, turtles, or crocodilians).

Index test (I): the technology of interest (sUAS).

Target condition $(\mathrm{T})$ : presence or abundance of population.

\section{Methods \\ Searching for articles \\ Search terms and languages}

All searches will be performed in English (i.e., search terms will only be in English), and only studies published in or translated to English will be included in this systematic map due to the languages understood by the systematic map team. Some search terms are specific to North America due to stakeholder driven requirements, but the geographic scope of studies returned or included in this systematic map will not be limited.

When applicable, search terms were truncated and a wildcard $\left({ }^{*}\right)$ added at the end of the root word to include all alternate forms of root words, in order to account for alternative spelling or hyphenation (e.g.,

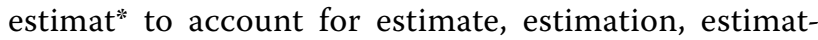
ing, estimates). Dollar signs (\$) were added when applicable to designate the addition of one extra character. Quotation marks were placed around some multiple word terms to allow for the search of exact phrases. All search terms and phrases were combined using the Boolean operators AND or OR. Additional terms for the three major groups will be added if found and deemed necessary from literature and reference searches. All searches will be conducted on the article title, abstract, and keywords with the exception of Google Scholar that only allows for whole text or title only searches.

\section{Search string}

We conducted a test search on October 20th 2020, in multiple databases, as part of a scoping exercise to help build search terms and ensure the correct use of operators to yield the best performance in returning results. An example search string that produced efficient results with $>99.99 \%$ accuracy among two searchers (1502 in Web of Science, 2041 in Scopus, 172 in Wildlife and 
Ecology Studies Worldwide, and 144 in Proquest Dissertations and Theses) is presented below:

[(UAS\$ OR UAV\$ OR UVS\$ OR RPA\$ OR "unmanned aerial system\$" OR "unmanned aerial vehicle\$" OR "unmanned vehicle system\$" OR "unmanned aircraft" OR "remotely piloted aircraft" OR "unoccupied aerial vehicle\$" OR "unoccupied aerial system\$" OR "drone\$")

\section{AND}

(animal" OR avian OR bird" OR mammal* OR reptil* OR wildlife OR carnivor* OR cattle OR deer OR furbearer* OR livestock OR mesocarnivore* OR shorebird* OR ungulate* OR waterbird* OR waterfowl* OR alligator* OR blackbird* OR cow* OR coyote* OR dove* OR eagle* OR geese OR goose OR gull* OR hawk* OR hog* OR owl* OR passeri* OR pig* OR quail* OR raptor* OR snake* OR starling* OR "terrestrial vertebrate\$” OR turkey* OR turtle* OR vulture*)

AND

(abundance* OR assess* OR count* OR estimat* OR monitor* OR population*)]

\section{Publication databases}

Relative to online publication databases, we will perform searches through Mississippi State University Libraries on the Web of Science search platform, Scopus, Wildlife and Ecology Studies Worldwide, and Proquest Dissertations and Theses. Databases included in the Web of Science search platform are the Core Collection, SciELO Citation Index, and Zoological Record. Databases were chosen based on the comprehensive coverage of published literature.

\section{Internet searches}

An internet search will be conducted using Google Scholar, and the first 100 or $10 \%$ results (whichever is fewer) sorted by relevance will be examined.

\section{Supplementary searches}

A search for non-peer reviewed literature will be conducted by request through a professional network of collaborators (e.g., United States Department of Agriculture, Federal Aviation Administration, United States Department of Defense, The Wildlife Society) and publicly available websites including Research Gate (http:// www.researchgate.net), LinkedIn (http://www.linkedin. com), Academia (http://www.academia.edu) and Twitter (http://twitter.com) as well as the Ecological Society of America mailing list (https://www.esa.org/membership/ ecolog/) (Additional file 2). Government reports, white papers, gray literature, and information from conference proceedings not returned from our primary searches will be included and undergo all screening. We will also scan reference lists of all articles included after full text screening for our aforementioned search terms, review articles, and "cited by lists" for the top- 20 most cited articles, to search for relevant, but missed articles which will then be added to the search results.

\section{Search settings}

To help control for bias injected into searches by learning algorithms of internet browsers, browser history and cookies will be disabled when conducting all searches. The search team will use "InPrivate" or "incognito" mode and will not access any electronic accounts. All searches will be conducted by two search team members to ensure a consistent number of returned articles.

\section{Comprehensiveness of the search}

To evaluate the comprehensiveness of the search strategy, we compiled a list of 41 benchmark articles (Additional file 3) from and including two recent reviews; we included articles listed under the "wildlife research and management" section of one review [33] and under the "UAS" section of another review [34]. Articles deemed outside the realm of our search (e.g., marine, nonsUAS, etc.) were excluded from our list of benchmark articles. We will compare the article records returned from our search to this list, and if any are found to be missing, the search string will be amended.

\section{Article screening and study eligibility criteria Screening process}

We will screen article records using a hierarchical approach in the order of title, abstract, and full text. Information for all article records returned from our search will first be imported to Rayyan to resolve duplicates and screen for relevance (i.e., those using UAS to monitor terrestrial animals) [35]. Any article records that cannot be deemed relevant or irrelevant will be included in the next stage of screening (i.e., records that cannot be determined as relevant at the title stage will move forward to the abstract stage of screening). A subset of 100 or $10 \%$ of article records (whichever is fewer) at each stage will be assessed by 2 reviewers, and we will calculate Cohen's kappa coefficient to determine agreement between reviewers, with $\mathrm{K} \geq 0.6$ indicating consistency. If inconsistency occurs, reviewers will discuss, clarify, and modify inclusion criteria as necessary and a new subset of article records will be reviewed, and reviewer agreement assessed. Any article records moving to the full text stage will be accessed using licenses through Mississippi State University, Google 
Scholar, U.S. Department of Agriculture, or personal inquiries with corresponding authors of articles. We will track article record exclusion stages and record exclusion reasonings in an Excel table and include this table in the systematic map as additional material.

\section{Eligibility criteria}

Eligible population: all terrestrial vertebrate animals including humans, wildlife, and domestic animals will be deemed as eligible subjects.

Eligible index test: any studies that use sUAS technology to monitor (i.e., identify, count, estimate, etc.) eligible populations will be included.

Eligible target condition: any study reporting presence or abundance of animal population will be included. Studies observing strictly behavior or deterrence, or opinion, comment, or discussion type manuscripts will be excluded from the systematic map.

Eligible study designs: any studies designed to count or monitor terrestrial vertebrate animals will be deemed as eligible studies. We will only evaluate studies published in or translated to English. We will not apply any date restrictions.

\section{Study validity assessment}

No formal study validity assessment will be conducted, but study design elements will be coded and reported.

\section{Data coding strategy}

Data extracted from studies will included a variety of aspects from categories including bibliographic information, study characteristics, index information, population information, and target information. See Additional file 4: Table S1 for a complete overview of our proposed data coding strategy. These data will initially be recorded in Microsoft Access to reduce redundancies in the database. The extracted information will be used to summarize whether sUAS can be used to monitor and count animals. If data from an article are confusing or not easily comprehensible, we will contact corresponding authors for clarification. Two reviewers will extract data from a subset of 100 or $10 \%$ of articles (whichever is fewer) to determine data accuracy of extracted information, again using kappa coefficients of $\mathrm{K} \geq 0.6$ to indicate consistency, and discussing, clarifying, and modifying data extraction criteria if necessary.

\section{Study mapping and presentation}

In the resulting systematic map, we will describe the process and include a summarized narrative and numbers of articles for each stage of the inclusion process and extracted data. We will publish the final database with all extracted data, both as an Excel spreadsheet and an Access database. Different queries from the final database will be used to produce tables, figures, and geographic maps, which will be accompanied and fully explained using a narrative review to answer our primary and secondary questions. Any additional subtopic or questions identified through the course of the systematic mapping process will be described in detail in the resulting systematic map. We will also create heat maps of study frequencies to identify major knowledge clusters and gaps which will both be discussed for future research. Finally, we will make recommendations for priorities in future research on using sUAS to monitor or count terrestrial vertebrate animals.

\section{Supplementary Information}

The online version contains supplementary material available at https://doi. org/10.1186/s13750-021-00228-w.

Additional file 1. Adherence to the ROSES guidelines.

Additional file 2. Call for supplementary literature.

Additional file 3. List of benchmark articles.

Additional file 4: Table S1. Data coding strategy spreadsheet.

\section{Acknowledgements}

We thank the Federal Aviation Administration and USDA APHIS for their support of this project and interest in advancing sUAS applications. We especially thank Dr. Wesley Major, Michael DiPilato, and Jonathan Sheairs for their review of this manuscript and associated materials. This publication is a contribution of the Mississippi State University Center for Resolving Human-Wildlife Conflicts, Forest and Wildlife Research Center, College of Forest Resources, and Geosystems Research Institute. Any opinions, findings, conclusions, or recommendations expressed in this publication are those of the author(s) and do not necessarily reflect the view of the US Department of Agriculture or Federal Aviation Administration policy decisions regarding sUAS or wildlife.

\section{Authors' contributions}

JAE drafted the manuscript and JAE and MFC conducted the scoping stage. All authors contributed to providing revisions on drafts, and read the final version of this systematic map protocol. All authors read and approved the final manuscript

\section{Funding}

This work was funded by US Department of Agriculture Animal and Plant Health Inspection Service (USDA APHIS; Cooperative Agreements AP20WSNWRC00C010 and AP20WSNWRC00C026) to Mississippi State University (R.B. Iglay, S. Samiappan, K. O. Evans) and an Interagency Agreement between USDA APHIS and the Federal Aviation Administration (FAA IA No. 692M1519-T-00017/Task Order No. 692M15-19-F-00348) for Task Order No. 2, Research Activities on Wildlife Hazards to Aviation. Additional support was provided by the Forest and Wildlife Research Center and College of Forest Resources at Mississippi State University.

\section{Declarations}

Availability of data and materials

Data sharing is not applicable as no datasets were generated nor analyzed for this systematic map protocol.

Ethics approval and consent to participate

Not applicable. 


\section{Consent for publication}

Not applicable.

\section{Competing interests}

The authors declare that they have no competing interests.

\section{Author details}

${ }^{1}$ Department of Wildlife, Fisheries and Aquaculture, Mississippi State University, Thompson Hall, Mississippi State, Mississippi 39762, USA. ${ }^{2}$ Geosystems Research Institute, Mississippi State University, Mississippi State, Mississippi 39762, USA. ${ }^{3}$ US Department of Agriculture, Animal and Plant Health Inspection Service, Wildlife Services, National Wildlife Research Center, Ohio Field Station, Sandusky, OH, USA.

Received: 17 December 2020 Accepted: 8 June 2021

Published online: 30 June 2021

\section{References}

1. Sullivan JM. Evolution or revolution? The rise of UAVs. IEEE Technol Soc Mag. 2006;25:43-9.

2. FAA. Summary of small unmanned aircraft rule (part 107). 2016. https:// www.faa.gov/uas/media/part_107_summary.pdf.

3. Anderson K, Gaston KJ. Lightweight unmanned aerial vehicles will revolutionize spatial ecology. Front Ecol Environ. 2013;11:138-46.

4. Christie KS, Gilbert SL, Brown CL, Hatfield M, Hanson L. Unmanned aircraft systems in wildlife research: current and future applications of a transformative technology. Front Ecol Environ. 2016;14:241-51.

5. Hodgson JC, Mott R, Baylis SM, Pham TT, Wotherspoon S, Kilpatrick AD, et al. Drones count wild life more accurately and precisely than humans. Methods Ecol Evol. 2018;9:1160-7.

6. Linchant J, Lisein J, Semeki J, Lejeune P, Vermeulen C. Are unmanned aircraft systems (UASs) the future of wildlife monitoring? A review of accomplishments and challenges. Mamm Rev. 2015:45:239-52.

7. Sasse DB. Job-related mortality of wildlife workers in the United States, 1937-2000. Wildl Soc Bull. 2003;31:1015-20.

8. Frederick PC, Hylton B, Heath JA, Ruane M. Accuracy and variation in estimates of large numbers of birds by individual observers using an aerial survey simulator. J F Ornithol. 2003;74:281-7.

9. Buckland ST, Burt ML, Rexstad EA, Mellor M, Williams AE, Woodward R. Aerial surveys of seabirds: the advent of digital methods. J Appl Ecol. 2012:49:960-7.

10. Chabot D, Bird DM. Wildlife research and management methods in the 21st century: where do unmanned aircraft fit in? J Unmanned Veh Syst. 2015:3:137-55.

11. Pimm SL, Alibhai S, Bergl R, Dehgan A, Giri C, Jewell Z, et al. Emerging technologies to conserve biodiversity. Trends Ecol Evol. 2015:30:685-96.

12. Hodgson JC, Baylis SM, Mott R, Herrod A, Clarke RH. Precision wildlife monitoring using unmanned aerial vehicles. Sci Rep. 2016;6:1-7.

13. Weinstein BG. A computer vision for animal ecology. J Anim Ecol. 2018;87:533-45

14. Reintsma KM, McGowan PC, Callahan C, Collier T, Gray D, Sullivan JD, et al. Preliminary evaluation of behavioral response of nesting waterbirds to small unmanned aircraft flight. Waterbirds. 2018:41:326-31.

15. Scholten CN, Kamphuis AJ, Vredevoogd KJ, Lee-Strydhorst KG, Atma $J$, Shea CB, et al. Real-time thermal imagery from an unmanned aerial vehicle can locate ground nests of a grassland songbird at rates similar to traditional methods. Biol Conserv. 2019;233:241-6.

16. Chabot D, Bird DM. Evaluation of an off-the-shelf unmanned aircraft system for surveying flocks of geese. Waterbirds. 2012;35:170-4.
17. Chabot D, Francis CM. Computer-automated bird detection and counts in high-resolution aerial images: a review. J F Ornithol. 2016;87:343-59.

18. Lyons MB, Brandis KJ, Murray NJ, Wilshire JH, McCann JA, Kingsford RT, et al. Monitoring large and complex wildlife aggregations with drones. Methods Ecol Evol. 2019;10:1024-35.

19. Guo X, Shao Q, Li Y, Wang Y, Wang D, Liu J, et al. Application of UAV remote sensing for a population census of large wild herbivores - taking the headwater region of the Yellow River as an example. Remote Sens. 2018;10:1041.

20. Hu J, Wu X, Dai M. Estimating the population size of migrating Tibetan antelopes Pantholops hodgsonii with unmanned aerial vehicles. Oryx. 2020;54:101-9.

21. Wich S, Dellatore D, Houghton M, Ardi R, Koh LP. A preliminary assessment of using conservation drones for Sumatran orang-utan (Pongo abelii) distribution and density. J Unmanned Veh Syst. 2016;4:45-52.

22. Bonnin N, Van Andel A, Kerby J, Piel A, Pintea L, Wich S. Assessment of chimpanzee nest detectability in drone-acquired images. Drones. 2018;2:17.

23. Thapa GJ, Thapa K, Thapa R, Jnawali SR, Wich SA, Poudyal LP, et al. Counting crocodiles from the sky: monitoring the critically endangered gharial (Gavialis gangeticus) population with an unmanned aerial vehicle (UAV). J Unmanned Veh Syst. 2018;6:71-82.

24. Rischette AC, Hovick TJ, Elmore RD, Geaumont BA. Use of small unmanned aerial systems for sharp-tailed grouse lek surveys. Wildl Biol. 2020. https://doi.org/10.2981/wlb.00679.

25. Kissell RE, Nimmo SK. A technique to estimate white-tailed deer Odocoileus virginianus density using vertical-looking infrared imagery. Wildl Biol. 2011;17:85-92.

26. Barnas AF, Chabot D, Hodgson AJ, Johnston DW, Bird DM, Ellis-Felege SN. A standardized protocol for reporting methods when using drones for wildlife research. J Unmanned Veh Syst. 2020;8:89-98.

27. Brack IV, Kindel A, Oliveira LFB. Detection errors in wildlife abundance estimates from unmanned aerial systems (UAS) surveys: synthesis, solutions, and challenges. Methods Ecol Evol. 2018;9:1864-73.

28. Anderson DR. The need to get the basics right in wildlife field studies. Wildl Soc Bull. 2001:29:1294-7.

29. James KL, Randall NP, Haddaway NR. A methodology for systematic mapping in environmental sciences. Environ Evid. 2016:5:1-13.

30. Mulero-Pázmány M, Jenni-Eiermann S, Strebel N, Sattler T, Negro JJ, Tablado Z. Unmanned aircraft systems as a new source of disturbance for wildlife: a systematic review. PLOS ONE. 2017;12:e0178448.

31. Lima SL, Blackwell BF, DeVault TL, Fernández-Juricic E. Animal reactions to oncoming vehicles: a conceptual review. Biol Rev. 2015;90:60-76.

32. Dolbeer RA, Beiger MJ, Miller PR, Weller JR, Anderson AL. Wildlife strikes to civil aircraft in the United States 1990-2019. Washington. 2021. https:// trid.trb.org/view/1853561.

33. Jiménez López J, Mulero-Pázmány M. Drones for conservation in protected areas: present and future. Drones. 2019;3:10

34. Wang $D$, Shao $Q$, Yue $H$. Surveying wild animals from satellites, manned aircraft and unmanned aerial systems (UASs): a review. Remote Sens. 2019;11:1308

35. Ouzzani M, Hammady H, Fedorowicz Z, Elmagarmid A. Rayyan-a web and mobile app for systematic reviews. Syst Rev. 2016;5:210.

\section{Publisher's Note}

Springer Nature remains neutral with regard to jurisdictional claims in published maps and institutional affiliations. 